\title{
Natural Frequencies of Human Corticothalamic Circuits
}

\author{
Mario Rosanova, ${ }^{1}$ Adenauer Casali, ${ }^{1}$ Valentina Bellina, ${ }^{1}$ Federico Resta, ${ }^{2}$ Maurizio Mariotti, ${ }^{1}$ and Marcello Massimini ${ }^{1}$ \\ ${ }^{1}$ Department of Clinical Sciences, "Luigi Sacco," Università degli Studi di Milano, and ${ }^{2}$ Division of Radiology, Ospedale Luigi Sacco, 20157 Milan, Italy
}

The frequency tuning of a system can be directly determined by perturbing it and by observing the rate of the ensuing oscillations, the so called natural frequency. This approach is used, for example, in physics, in geology, and also when one tunes a musical instrument. In the present study, we employ transcranial magnetic stimulation (TMS) to directly perturb a set of selected corticothalamic modules (Brodmann areas 19,7, and 6) and high-density electroencephalogram to measure their natural frequency. TMS consistently evoked dominant $\alpha$-band oscillations $(8-12 \mathrm{~Hz}$ ) in the occipital cortex, $\beta$-band oscillations $(13-20 \mathrm{~Hz})$ in the parietal cortex, and fast $\beta / \gamma$-band oscillations $(21-50 \mathrm{~Hz})$ in the frontal cortex. Each cortical area tended to preserve its own natural frequency also when indirectly engaged by TMS through brain connections and when stimulated at different intensities, indicating that the observed oscillations reflect local physiological mechanisms. These findings were reproducible across individuals and represent the first direct characterization of the coarse electrophysiological properties of three associative areas of the human cerebral cortex. Most importantly, they indicate that, in healthy subjects, each corticothalamic module is normally tuned to oscillate at a characteristic rate. The natural frequency can be directly measured in virtually any area of the cerebral cortex and may represent a straightforward and flexible way to probe the state of human thalamocortical circuits at the patient's bedside.

\section{Introduction}

The spontaneous waking electroencephalogram (EEG) suggests that different regions of the human brain tend to engage in electrical oscillations at different frequencies (Niedermeyer, 1999). For example, when a subject lays eyes closed in a state of relaxation, occipital areas typically oscillate at a frequency of $\sim 10 \mathrm{~Hz}$ ( $\alpha$ rhythm), whereas sensory-motor cortex often displays faster rhythms, at $\sim 20 \mathrm{~Hz}$ ( $\beta$ rhythm). Yet, spontaneous EEG rhythms are rather variable, and their topography can change radically in the space of a second if the subject, for instance, opens his eyes or plans a simple movement. Hence, it is difficult to tell, from the spontaneous EEG alone, whether different cortical circuits are intrinsically tuned to generate oscillations at any particular frequency.

A more reliable estimate of the frequency tuning of some cortical areas can be obtained by measuring steady-state-evoked responses. In this case, visual flashes, or auditory tones, are presented at different rates, and the stimulation frequency that results in the largest EEG or magnetoencephalography output, the resonance frequency, is detected. This standardized approach yielded consistent results and demonstrated the existence of clear-cut resonance frequencies in specific parts of the human corticothalamic system, $\sim 10 \mathrm{~Hz}$ (Narici and Romani, 1989; Regan, 1989; Silberstein, 1995; Rager and Singer, 1998; Herrmann, 2001) in visual cortex and $\sim 40 \mathrm{~Hz}$ in auditory cortex

Received Jan. 27, 2009; revised April 10, 2009; accepted April 12, 2009.

This work was supported by European Grants Strep LSHM-CT-2205-51818, PRIN 2006, and EU-Grant-224328PredictAD (to M. Massimini). We thank Giulio Tononi, Steve Esser, Brady Riedner, Fabio Ferrarelli, and Silvia Casarotto for their help and comments.

Correspondence should be addressed to Marcello Massimini, Department of Clinical Sciences "Luigi Sacco," Via GB Grassi 74, 20157 Milan, Italy. E-mail: marcello.massimini@unimi.it.

D01:10.1523/JNEUROSC1.0445-09.2009

Copyright $\odot 2009$ Society for Neuroscience $\quad$ 0270-6474/09/297679-07\$15.00/0
(Galambos et al., 1981; Pastor et al., 2002). However, steady-state responses, as other responses evoked by the stimulation of peripheral receptors, can only probe a limited set of primary sensory cortices. Moreover, they assess the tuning of corticothalamic circuits only indirectly, since they can be significantly affected by the bandwidth of peripheral receptors (Abraham and Alpern, 1984) by sensory gating (Emerson et al., 1988), attention levels (Hillyard et al., 1997), and stimulus perception (Tononi et al., 1998; Cosmelli et al., 2004).

In principle, a straightforward way of probing the frequency tuning of a system is to directly perturb it to detect the main rate of the ensuing oscillations, the so called natural frequency. This approach is commonly used in physics and geology but also when one tunes a musical instrument or, instinctively, knocks on the surface of an object to appreciate its internal structure. Indeed, measuring the natural frequency may reveal important information about the structure, the properties, and the state of the system under study. In this work, we used transcranial magnetic stimulation (TMS) to perturb directly different regions of the human corticothalamic system (area 19, area 7, and area 6) and high-density EEG (hd-EEG) to measure their natural frequency.

\section{Materials and Methods}

Subjects. Seven right-handed subjects (four females, age 23-37) participated in this study. The experimental procedures were approved by the local ethical committee of the Hospital Luigi Sacco, Università degli Studi di Milano. All participants gave written informed consent and underwent a neurological screening to prevent potential adverse effects of TMS. Each subject was invited for three separate experiments. During each experiment, we targeted one of three cortical sites (occipital, parietal, and frontal), and we performed several (6-8) TMS/hd-EEG sessions at different stimulation intensities. During a single TMS/hd-EEG session, we collected 100-200 trials. Data from one subject were excluded, because of a low signal-to-noise ratio of TMS/EEG-evoked responses. In total, $150 \mathrm{TMS} / \mathrm{hd}-\mathrm{EEG}$ sessions were performed, 120 of which were used for this study. 
Brain-navigated TMS. A focal bipulse, figure-of-eight coil with $60 \mathrm{~mm}$ wing diameter driven by a biphasic stimulator (eXimia TMS Stimulator; Nexstim) was used to stimulate the subjects' cortex. Three cortical sites (middle or superior occipital gyrus, superior parietal gyrus, and middle or caudal portion of the superior frontal gyrus) were selected based on an atlas of brain regional anatomy (Tamraz and Comair, 2000), anatomically identified on a T1-weighted individual MRI (resolution $1 \mathrm{~mm}$ ) acquired with a $1 \mathrm{~T}$ Philips scanner and were targeted by means of a Navigated Brain Stimulation (NBS) system (Nexstim). The NBS is able to locate (error $<3 \mathrm{~mm}$ ) the relative positions of the subject's head and the TMS coil by means of an optical-tracking system. The NBS takes into account the individual's head shape, the coil position, and scalp-tocortex distance to calculate the electric field induced by TMS on the cortical surface. During the experiments, we adjusted TMS intensity according to the maximum electric field intensity (expressed in volts per meter) estimated on the cortical surface, rather than relying on individual motor threshold or on the percentage of maximum stimulator output. This procedure ensured that all cortical areas, in all subjects, were stimulated at comparable intensities, despite large differences in scalpto-cortex distance and anatomy. To further standardize stimulation parameters, the maximum electric field was always kept on the convexity of the gyrus with the induced current perpendicular to its main axis. Each cortical area was stimulated at 20-40-60-80-100-120-140-160 V/m. The coordinates of the stimulator were input to a virtual aiming device of the navigation software and used during the experiment to ensure stability of the position, angle, direction, and intensity of the stimulation. At the end of each experimental session, EEG sensor positions were digitized by means of a pen visible to the optical-tracking system and stored in the navigation computer.

High-density EEG recordings during TMS. We recorded TMS-evoked potentials using a TMS-compatible 60-channel amplifier (Nexstim) which gates the TMS artifact and prevents saturation by means of a proprietary sample-and-hold circuit (Virtanen et al., 1999). The impedance at all electrodes was kept $<5 \mathrm{k} \Omega$. The EEG signals, referenced to an additional electrode on the forehead, were filtered $(0.1-500 \mathrm{~Hz})$ and sampled at $1450 \mathrm{~Hz}$ with 16-bit resolution. Two extra sensors were used to record the electrooculogram. In most cases, no TMS-induced magnetic artifacts were detected, and in all cases, the EEG signals were artifact-free starting from $8 \mathrm{~ms}$ after stimulus. To avoid contamination of TMS-evoked potential by auditory potentials evoked by the click associated with the TMS discharge, subjects were wearing inserted earplugs continuously playing a masking noise capturing the specific time-varying frequency components of the TMS click. Bone conduction was attenuated by placing a thin layer of foam between coil and scalp. These procedures have been previously shown to effectively abolish the auditory potentials evoked by the TMS-associated clicks (Massimini et al., 2005, 2007; Ferrarelli et al., 2008). Other possible sources of artifacts or extracranial sources were controlled and excluded during off-line analysis (see supplemental Appendix B, available at www.jneurosci.org as supplemental material; Figs. 2-4)

General experimental procedures. During the experiment, subjects were lying on an ergonomic chair, relaxed, and with eyes open looking at a fixation point on a screen. A headrest ensured a comfortable and stable head position. Once the selected cortical site was targeted, we stimulated it at different intensities. For each intensity, we delivered between 100 and 200 stimuli at a frequency jittering between $0.4-0.5 \mathrm{~Hz}$ (period, 2000 plus a jitter $\leq 300 \mathrm{~ms}$ ). All stimulation parameters were set according to international safety guidelines (Wassermann, 1998).

Data analysis and statistics. Data analysis was performed using Matlab R2006a (The MathWorks). TMS trials containing noise, muscle activity, or eye movements were automatically detected and rejected. EEG data were average referenced, down sampled at half of the original sampling rate $(725 \mathrm{~Hz})$, and bandpass filtered $(2-80 \mathrm{~Hz})$. Source modeling was performed by applying an "empirical" Bayesian approach to single trials (for more details, see supplemental Appendix B, available at www. jneurosci.org as supplemental material).

To analyze the responses in the time-frequency domain, both at the sensor and at the source level, we calculated the event-related spectral perturbation (ERSP) based on Morlet wavelets. The procedure was im- plemented using the public license toolbox EEGLAB (Delorme and Makeig, 2004) (for more details about ERSP calculation, see supplemental Appendix B, available at www.jneurosci.org as supplemental material). The global ERSP (gERSP) was computed by averaging the ERSPs of all channels in a given session. To minimize the effect of possible artifacts occurring at the time of stimulation, the natural frequency was calculated by averaging the ERSP values in a time window between 20 and $200 \mathrm{~ms}$.

\section{Results}

Three different cortical areas (Brodmann areas 19, 7, and 6) were targeted by TMS during three separated experiments in six healthy subjects. Using the MRI-guided navigation system, we stimulated each cortical area at eight different intensities (range, $20-160 \mathrm{~V} / \mathrm{m}$ ). EEG responses were recorded by means of a TMScompatible 60-channel amplifier.

\section{The main frequency of TMS-evoked EEG oscillations depends on the site of stimulation}

Figure 1 shows the EEG responses recorded in one subject from all sensors after stimulation of area 19, area 7, and area 6 (the stimulation hot-spot is marked by a red dot on the individual MRI) are displayed. In this example, TMS was delivered at an electric field intensity of $120 \mathrm{~V} / \mathrm{m}$ on the cortical surface, a stimulation level that is known to be above motor threshold (Epstein et al., 1990; Thielscher and Kammer, 2002). Superimposing the averaged TMS-evoked potentials recorded from all channels in a butterfly plot revealed that the brain electrical response to TMS varied markedly depending on the site of stimulation: TMS of area 19 resulted, during the first $200 \mathrm{~ms}$, in a low-frequency, large response; stimulation of area 7 elicited faster and smaller components; stimulation of area six evoked the fastest EEG oscillations. To detect the frequency of the oscillations induced by TMS at different cortical sites, we calculated the ERSP. The ERSP decomposes the EEG response recorded from each sensor in the timefrequency space and allows tracking the significant spectral modulations induced by TMS during the poststimulus time (see Materials and Methods; supplemental Appendix C, available at www.jneurosci.org as supplemental material). As a first step, to study the main frequency of the overall brain response to TMS, we averaged, for each condition, the ERSPs matrices across all channels, thus obtaining a gERSP. The bottom panels of Figure 1 display the resulting plots for the three stimulation sites. In all cases, TMS resulted in a significant early $(8-20 \mathrm{~ms})$ activation in the $\beta 2 / \gamma$-bands $(21-50 \mathrm{~Hz})$. After this short-lasting stereotypical event, the frequency content of the global brain response to TMS varied markedly, depending on the stimulated area: dominant oscillations in the $\alpha$ range $(8-12 \mathrm{~Hz})$ were detected when area 19 was stimulated, in the $\beta 1$ range $(13-20 \mathrm{~Hz})$ when area 7 was stimulated, and in the $\beta 2 / \gamma$ range $(21-50 \mathrm{~Hz})$ when area 6 was stimulated. The same results were obtained whether subjects were instructed to keep their eyes open or closed (see supplemental Appendix A, available at www.jneurosci.org as supplemental material; Fig. 1). To extract the natural frequency of the brain's response in each condition, we averaged the gERSP over the first $200 \mathrm{~ms}$ after stimulus, and we detected, on the resulting spectral profiles (black trace on the right of each panel), the frequency with maximum power (indicated by a dotted line). Across all subjects, at the intensity of $120 \mathrm{~V} / \mathrm{m}$, the natural frequency of the global scalp response to stimulation of area 19 , area 7 , and area 6 was $11 \pm 1.5 \mathrm{~Hz}, 18.3 \pm 2.0 \mathrm{~Hz}$, and $29 \pm 2.0$, respectively (for individual values, see supplemental Table 1, available at www. jneurosci.org as supplemental material). Thus, TMS induced widespread scalp EEG oscillations with a dominant frequency that was characteristic for the stimulated site. 

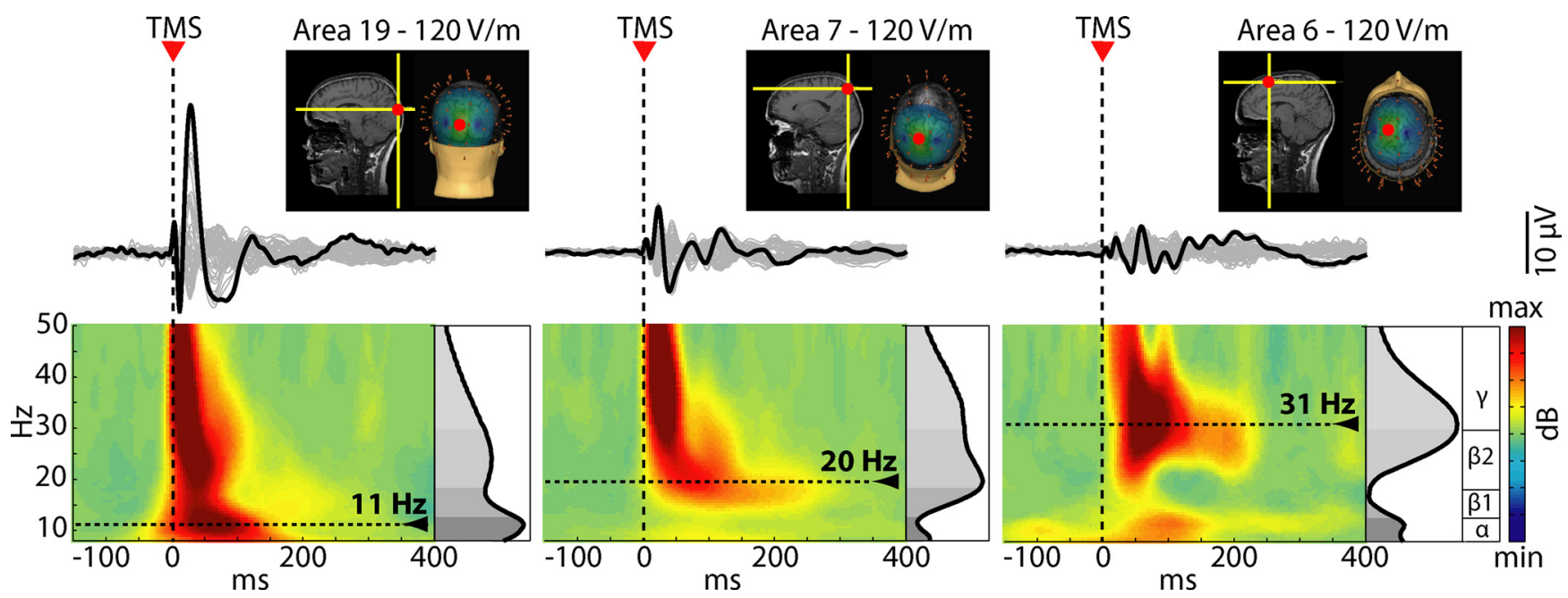

Figure 1. TMS induces global EEG oscillations that are specific for the stimulated site. The insets illustrate the three cortical sites targeted by TMS (hot spot on the individual MRI) in one subject. The traces below represent butterfly plots, where the black trace highlights the electrode directly underlying the stimulator. The bottom panels show the ERSP patterns calculated globally on the scalp (average of all electrodes). The gray scale graph plotted at the right of each ERSP depicts the power spectrum profile induced during the first $200 \mathrm{~ms}$ after TMS. The dotted lines highlight the frequency with maximum power, the natural frequency. TMS elicited early $\gamma$ components immediately followed by prominent $\alpha$-band oscillations after occipital stimulation, $\beta$-band oscillations after parietal stimulation, and fast $\beta / \gamma$ oscillations after perturbation of frontal cortex.

\section{The natural frequency reflects local properties of} corticothalamic circuits

As a next step, we wanted to minimize volume conduction effects to explore TMS-evoked oscillations on a finer spatial scale. To this aim, we performed source modeling of TMS-evoked potentials. We modeled individual cortical surfaces with a mesh of 7004 fixed dipoles, and we estimated local cortical currents using a Bayesian approach (see Materials and Methods). After automatic classification of the individual's cortical surface, each dipole was assigned to 1 of 47 Brodmann's areas, and time series of local TMS-evoked activation were obtained by cumulating the dipole activities recorded in each cortical area (see Materials and Methods). At this point, the same time-frequency decomposition analysis performed at the global sensors level was also performed at the local source level. Figure 2 shows the time series of the local current and the ERSP plots recorded from all three cortical areas of interest (area 19, area 7, and area 6) when TMS was directly applied to each of them. The large oscillations expressed locally by each cortical area when directly stimulated (Fig. 2, see the ERSP plots marked by the TMS icons) matched the dominant frequency recorded globally at the scalp level (Fig. 1). On the other hand, even when not directly stimulated, each cortical area still tended to oscillate at a rate closer to its own natural frequency. Across all subjects, when area 19 was stimulated, area 19 responded at $10.8 \mathrm{~Hz}$, area 7 at $20 \mathrm{~Hz}$, and area 6 at $31.3 \mathrm{~Hz}$; when area 7 was stimulated, area 19 responded at $13.5 \mathrm{~Hz}$, area 7 at 18.6 $\mathrm{Hz}$, and area 6 at $27.3 \mathrm{~Hz}$; when area 6 was stimulated, area 19 responded at $10.6 \mathrm{~Hz}$, area 7 at $19 \mathrm{~Hz}$, and area 6 at $29 \mathrm{~Hz}$ (for SDs and ranges, see Table 1; for individual values, see supplemental Table 2, available at www.jneurosci.org as supplemental material). Hence, each area, whether directly activated by TMS or engaged through long-range connections, expressed local oscillations at a rate closer to its own natural frequency.

The local natural frequency is preserved across a wide range of stimulation intensities

The characteristics of the EEG response to TMS may depend on the intensity of stimulation (Komssi et al., 2004; Kähkönen et al., 2005a), and different corticothalamic modules may have differ- ent thresholds and reactivity to TMS (Kähkönen et al., 2005b). Thus, the site-specificity of the natural frequency may be an intensity-dependent phenomenon and may be lost at high stimulation intensities. To exclude this possibility, we stimulated each cortical site at several increasing intensities, from subthreshold level to saturation of the averaged EEG response. For each cortical area, we then calculated the input/output curves of the TMSinduced ERSP in four EEG frequency bands $(\alpha, 8-12 \mathrm{~Hz} ; \beta 1$, $13-20 \mathrm{~Hz} ; \beta 2,21-29 \mathrm{~Hz}$; and $\gamma, 30-50 \mathrm{~Hz}$ ). Figure 3 shows the three-dimensional (time, power, intensity) plots obtained for each frequency band in each stimulated area. TMS intensities $<40 \mathrm{~V} / \mathrm{m}$ did not result in any significant power modulation in any of the four EEG bands. Intensities of stimulation between 60 and $120 \mathrm{~V} / \mathrm{m}$ evoked progressively larger responses in all four frequency bands. At this level, the responses of the three corticothalamic modules clearly differed, displaying higher $\alpha$-band power after stimulation of area 19, higher $\beta 1$-band power after stimulation of area 7 , and higher $\beta 2 / \gamma$-band power after stimulation of area 6 . These differences further increased at stimulation intensities $(140-160 \mathrm{~V} / \mathrm{m})$ that produced saturation of the TMSevoked potentials. Hence, the site-specificity of the natural frequencies is not attributable to local differences in the ability of TMS to excite cortical neurons.

\section{Corticothalamic natural frequencies are reproducible across subjects}

Figure $4 A$ shows the evoked responses recorded from a sensor under the coil in all subjects at all intensities (superimposed traces). The black thinner traces represent the TMS-evoked responses obtained by stimulating at the maximal intensity (160 $\mathrm{V} / \mathrm{m}$ ). In all subjects, provided that TMS intensity was above the threshold for triggering a significant EEG response, the average waveform differed according to the site of stimulation. Occipital stimulation triggered high-amplitude, lower-frequency components, whereas stimulating more rostral sites elicited smaller waves separated by shorter intervals. TMS at $160 \mathrm{~V} / \mathrm{m}$ of area 19 elicited larger responses (first deflection in PO3, $48.4 \pm 12.8 \mu \mathrm{V}$ ) than an equivalent stimulation of area 7 and 6 (respectively, CP1: $13.8 \pm 5.21$ and FC1: $18.8 \pm 10.7$ ) (Wilcoxon rank test, $p<0.01$ ). 
The grand averages (thick traces at the bottom) calculated across all subjects (TMS at $160 \mathrm{~V} / \mathrm{m}$ ) highlight the reproducibility of the site-specificity of the waveforms. Figure $4 B$ shows the natural frequency calculated at the global sensor level in all subjects after stimulation of area 19, area 7, and area 6 at $160 \mathrm{~V} / \mathrm{m}$. In all cases, the natural frequency progressively increases from $\alpha$, to $\beta$, and $\gamma$ when the three sites are plotted in the caudal-rostral order. Thus, in healthy and awake subjects, each cortical area is normally tuned to oscillate at a preferred rate.

\section{Discussion}

The electrical oscillations evoked in the human brain by the direct stimulation of different cortical sites have never been studied and compared so far. Although we know that stimulating peripheral receptors of different sensory systems results in evoked potentials with specific latencies, waveforms, and spectral components (Niedermeyer, 1999), it is difficult to predict what would happen in the case of direct cortical perturbations. For instance, one might expect that the basic electrophysiological properties of the cerebral cortex are isotropic and that stimulating the cortex at different sites may result in the same stereotypical response. Our analysis at the EEG sensor level clearly indicated that, at least in the brain of healthy and awake subjects, this is not the case. Following an early (0-20 ms) stereotypical sharp component, TMS consistently evoked dominant $\alpha$-band oscillations after stimulation of the occipital cortex (area 19), $\beta$-band oscillations after stimulation of the parietal cortex (area 7 ), and fast $\beta / \gamma$ band oscillations after stimulation of the frontal cortex (area 6). This phenomenon originated intracranially (see supplemental Appendix B, available at www.jneurosci.

org as supplemental material; Figs. 2-4) and was immediately evident when the global TMS-evoked potential, recorded from all electrodes, was considered (Fig. 1). As subsequently revealed by sourcemodeling analysis, the dominant frequency recorded globally over the scalp mainly reflected the frequency of the oscillations generated by TMS in the stimulated area (Fig. 2). These sources, directly activated by TMS, generated strong currents that dominated, possibly because of volume conduction, the global EEG response recorded at the sensors' level.

TMS is known to evoke electrical activations not only at the stimulated site (Paus et al., 2001) but also in distant cortical regions (Ilmoniemi et al., 1997; Massimini et al., 2005). Hence, we asked what kind of activity is triggered by TMS in cortical areas that are not directly stimulated. Are distant, but connected, cortical circuits fully entrained by the strong oscillations triggered in the stimulated area, or do they tend to oscillate around their own preferred frequency? Reducing the effect of volume conduction with source modeling revealed that distant areas, when signifi- cantly activated, responded with oscillations closer to their own natural frequency (see supplemental Table 2, available at www. jneurosci.org as supplemental material). The ability of distant, yet connected, cortical oscillators to interact by using different frequencies has been suggested already (Friston, 1997; von Stein et al., 2000); this idea is corroborated by our experiments. Indeed, the same TMS pulse set in motion different connected oscillators, generating a complex EEG response composed of strong fluctuations at the natural frequency of the stimulated area and by weaker fluctuations at around the natural frequency of distant regions. More importantly, this particular finding indicates that the observed oscillations are not the para-physiological effect of nonecological magnetic brain activation but rather physiological events that can be engaged through brain connections. As recently demonstrated, during non-rapid eye movement sleep, the electrical oscillations evoked by TMS become slower and more stereotypical (Massimini et al., 2005, 2007). Hence, it will be interesting to test whether the site-specificity of the natural fre- 
Table 1. Mean values, SD, and range of the natural frequencies measured (across all subjects) in Brodmann areas 19, 7, and 6 when directly stimulated (bold) and when not directly stimulated

\begin{tabular}{lcr}
\hline & TMS area 19 & TMS area 7 \\
\hline $\begin{array}{l}\text { Area } 6 \\
\quad \text { Mean } \pm \text { SD (min-max) }\end{array}$ & $31.3 \pm 3.7$ & $25.6 \pm 6.5(17-33)$ \\
$\begin{array}{l}\text { Area } 7 \\
\quad \text { Mean } \pm \text { SD (min-max) }\end{array}$ & $20 \pm 4.8(12-25)$ & $\mathbf{2 9} \pm \mathbf{2 . 8}(\mathbf{2 6 - 3 4 )}$ \\
$\begin{array}{l}\text { Area } 19 \\
\quad \text { Mean } \pm \text { SD (min-max) }\end{array}$ & $\mathbf{1 8 . 6} \mathbf{3 ( 1 6 - 2 4 )}$ & $13.5 \pm 4.7(9-20)$ \\
\hline
\end{tabular}

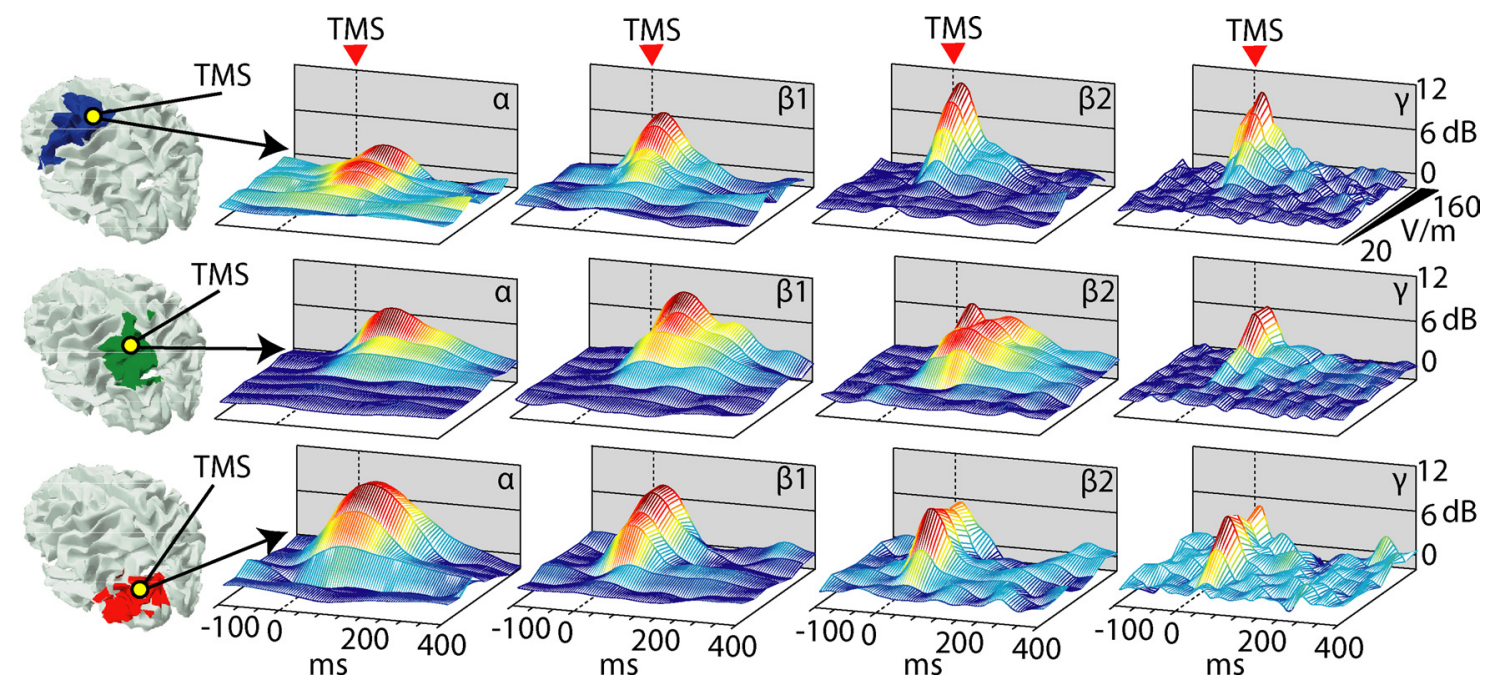

Figure 3. Different natural frequencies, in different cortical areas, are not attributable to different stimulation intensities. Time series (ERSP) of standard EEG frequency bands ( $\alpha, 8-12 ; \beta 1$, $13-20 ; \beta 2,21-29 ; \gamma, 30-50$ ) for the three sites of stimulation (first row, area 6; second row, area 7; third row, area 19) are plotted as a function of stimulation intensity, ranging from 20 to $160 \mathrm{~V} / \mathrm{m}$. The specificity of the natural frequency is preserved also when stimulation intensity approaches the saturation level.

quency is preserved in states where brain function is globally altered, such as during sleep, anesthesia, and in patients with disorders of consciousness.

An important parameter that may potentially bias the frequency of the EEG response to TMS is the strength of the applied stimulus. We used an MRI-guided navigation system to ensure that the strength of the electric field induced by TMS in different areas was always comparable. Moreover, to exclude an effect of local activation thresholds in determining the observed sitespecificity of the response's frequency, we probed each site at several different intensities, from subthreshold levels to nearsaturation levels. As shown in Figure 3, across this entire range, we detected higher power in the $\alpha$-band in the occipital cortex, in the $\beta$-band in the parietal cortex, and the fast $\beta / \gamma$-band in the frontal cortex. Thus, the specific frequency of the response did not depend on stimulation intensity, or activation threshold, but most likely depended on a number of endogenous properties of the activated circuit.

TMS stimulates patches of cortex of few square centimeters, and EEG source modeling has a spatial resolution of the same order. Thus, the natural frequencies defined with TMS/hd-EEG do not necessarily concern the fine grain physiological behavior of single neurons or minicolumns. Indeed, the electrical rhythms triggered by TMS are more likely to reflect overall circuit properties at the level of whole cortical areas and connected thalamic/ subcortical nuclei. For instance, a recent study has linked $\alpha$ oscillations to the presence of a subpopulation of electrically coupled neurons localized in the lateral geniculate nucleus which fire bursts of action potentials in the $\alpha$ range when activated by a cortical glutamatergic input (Hughes et al., 2004). This mechanism, involving a whole corticothalamic module, may explain why TMS of visual cortex readily triggers $\alpha$ oscillations, whereas TMS of frontal cortex fails to do so. Similarly, a role of the thalamus can be postulated in the genesis of faster oscillations (Llinás et al., 2007). Interestingly, TMS/EEG experiments performed in patients with lesions in the ventrolateral thalamus have demonstrated a significant decrease of $\beta$-band oscillations after TMS of the ipsi-lesional motor cortex (Van Der Werf et al., 2006).

The correlation between different EEG rhythms and the activity in different regions of the human brain has been known for long time. Almost a century ago, Adrian and Matthews (1934) reported that prominent EEG oscillations in the $\alpha$-band were observable at occipital sites. Subsequently, topographic differences of EEG rhythms have been further explored by means of source modeling (Michel et al., 1992; Gómez et al., 2006; Tinguely et al., 2006; Gómez-Herrero et al., 2008). More recently, combined EEG-functional MRI (fMRI) recordings performed in the resting condition detected a stronger correlation between $\alpha$ rhythm and occipital cortical activity (Laufs et al., 2003; Feige et al., 2005; Mantini et al., 2007) and between $\beta$ rhythm and parietal cortex activity (Laufs et al., 2003). A more recent EEG-fMRI study, using independent component analysis, also suggested a significant association between frontal cortex activity and $\gamma$ rhythm (Mantini et al., 2007). However, observing the ongoing EEG, although suggestive, is not enough to causally link individual cortical areas to specific rhythms. Spontaneous EEG activity, even in standardized conditions, is difficult to control and is not stable over time. At any given moment, some areas may be silent, 


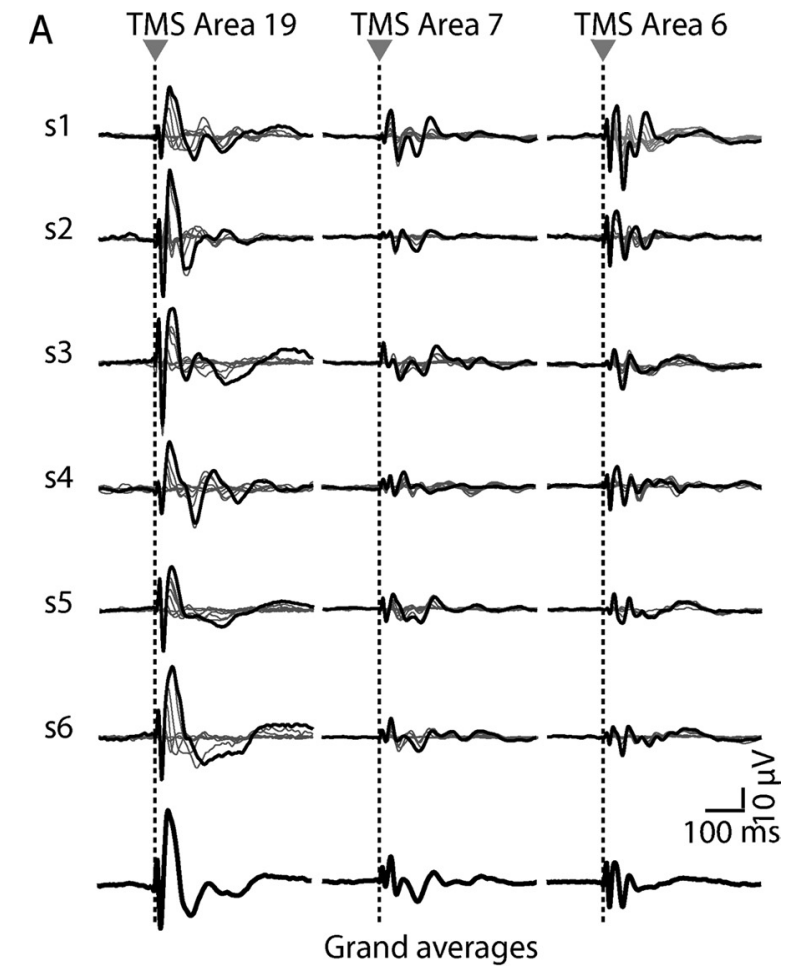

B

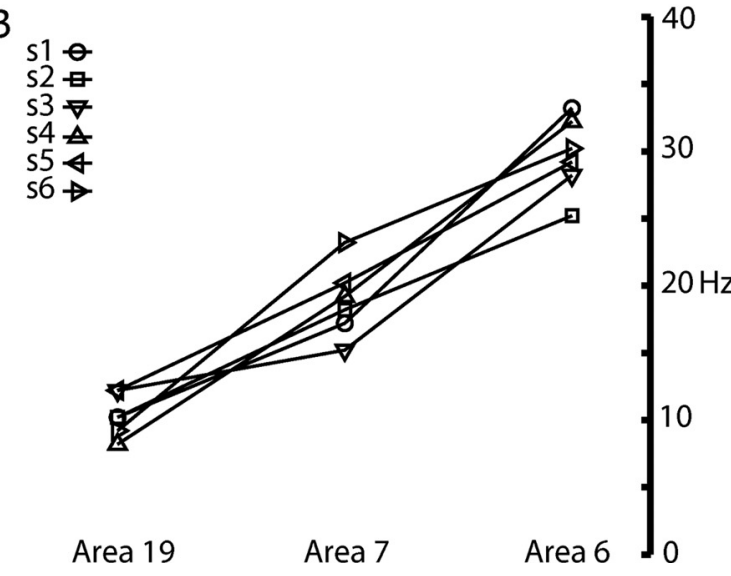

Figure 4. The specificity of the natural frequency is reproducible across subjects. $A$, The traces represent TMS responses for each subject $(n=6)$ evoked by $6-8$ intensities of stimulation, recorded from one electrode underlying the stimulator. The black trace highlights the response obtained at maximal stimulation intensity $(160 \mathrm{~V} / \mathrm{m})$. The thicker black traces at the bottom represent the grand averages calculated from all six subjects. $\boldsymbol{B}$, The frequencies with maximum power, obtained by stimulating each cortical area at the maximal intensity, are plotted for each subject. A clear posterior-anterior gradient of increasing frequencies is observable.

some other may be passively driven by other structures, and some may be engaged in specific patterns of activity depending on sensory inputs or cognitive state. In principle, the only way to know what a given cortical circuit can do, by virtue of its intrinsic properties and connections, is to directly and selectively activate it and measure its output. In this perspective, the approach used in the present study offers a promising tool to disentangle and study the local mechanisms that underlie the generation of compound EEG oscillations in humans. Thus, using controlled perturbations to identify the local frequency tuning of selected regions of the corticothalamic system, and detecting their interactions at the whole-brain level, represents an effective way to understand how brain rhythms arise and coalesce.
Moreover, detecting the natural frequency with TMS/hd-EEG may have diagnostic potential and clinical applications. Alterations in the membrane properties of subsets of cortical and subcortical (especially thalamic) neurons, as well as alterations in their patterns of connectivity, underlie most neurological and psychiatric conditions and may result in distinctive and detectable changes in their oscillatory properties (Soininen et al., 1992; Llinás et al., 1999, 2007; Jeong, 2004; Hughes and Crunelli, 2005). These alterations may be diffuse or local; thus, it will be important to map the natural frequency of different cortical areas in different neuropsychiatric conditions, such as depression, epilepsy, and disorders of consciousness. In a recent study, for example, we have demonstrated that the frontal cortex of schizophrenia patients, at difference with the one of healthy controls, fails to respond to TMS with prominent $\beta / \gamma$ EEG oscillations (Ferrarelli et al., 2008). Figure 4 summarizes the results obtained when measuring the natural frequency of area 19 , area 7 , and area 6 in six healthy subjects. These measurements represent the first direct exploration of the intrinsic electrophysiological properties of three associative areas of the human cerebral cortex. In addition, they provide a preliminary definition of normative values and clearly indicate that, in healthy and awake subjects, each one of the stimulated cortical areas is normally tuned to oscillate at a preferred rate. If the natural frequency reflects relevant physiological properties, TMS-evoked potentials may radically extend the window opened by peripherally evoked potentials. Indeed, whereas the latter are confined to the examination of sensory pathways and cortices, TMS/hd-EEG by-passes sensory, motor pathways, and subcortical structures. In this way, TMS-evoked potentials can access any cortical area (primary and associative), in any category of patients (including deafferented and locked-in subjects) and may offer a straightforward and flexible way to detect and monitor the state of corticothalamic circuits at the patient's bedside.

\section{References}

Abraham FA, Alpern M (1984) Factors influencing threshold of the fundamental electrical response to sinusoidal excitation of human photoreceptors. J Physiol 357:151-172.

Adrian E, Matthews B (1934) The Berger rhythm: potential changes from the occipital lobes in man. Brain 57:355-385.

Cosmelli D, David O, Lachaux JP, Martinerie J, Garnero L, Renault B, Varela F (2004) Waves of consciousness: ongoing cortical patterns during binocular rivalry. Neuroimage 23:128-140.

Delorme A, Makeig S (2004) EEGLAB: an open source toolbox for analysis of single-trial EEG dynamics including independent component analysis. J Neurosci Methods 134:9-21.

Emerson RG, Sgro JA, Pedley TA, Hauser WA (1988) State-dependent changes in the N20 component of the median nerve somatosensory evoked potential. Neurology 38:64-68.

Epstein CM, Schwartzberg DG, Davey KR, Sudderth DB (1990) Localizing the site of magnetic brain stimulation in humans. Neurology 40:666-670.

Feige B, Scheffler K, Esposito F, Di Salle F, Hennig J, Seifritz E (2005) Cortical and subcortical correlates of electroencephalographic alpha rhythm modulation. J Neurophysiol 93:2864-2872.

Ferrarelli F, Massimini M, Peterson MJ, Riedner BA, Lazar M, Murphy MJ, Huber R, Rosanova M, Alexander AL, Kalin N, Tononi G (2008) Reduced evoked gamma oscillations in the frontal cortex in schizophrenia patients: a TMS/EEG study. Am J Psychiatry 165:996-1005.

Friston KJ (1997) Another neural code? Neuroimage 5:213-220.

Galambos R, Makeig S, Talmachoff PJ (1981) A 40-Hz auditory potential recorded from the human scalp. Proc Natl Acad Sci U S A 78:2643-2647.

Gómez CM, Marco-Pallarés J, Grau C (2006) Location of brain rhythms and their modulation by preparatory attention estimated by current density. Brain Res 1107:151-160.

Gómez-Herrero G, Atienza M, Egiazarian K, Cantero JL (2008) Measuring directional coupling between EEG sources. Neuroimage 43:497-508. 
Herrmann CS (2001) Human EEG responses to 1-100 Hz flicker: resonance phenomena in visual cortex and their potential correlation to cognitive phenomena. Exp Brain Res 137:346-353.

Hillyard S, Hinrichs H, Tempelmann C, Morgan S, Hansen J, Scheich H, Heinze H-J (1997) Combining steady-state visual evoked potentials and fMRI to localize brain activity during selective attention. Hum Brain Mapp 5:287-292.

Hughes SW, Crunelli V (2005) Thalamic mechanisms of EEG alpha rhythms and their pathological implications. Neuroscientist 11:357-372.

Hughes SW, Lörincz M, Cope DW, Blethyn KL, Kékesi KA, Parri HR, Juhász G, Crunelli V (2004) Synchronized oscillations at alpha and theta frequencies in the lateral geniculate nucleus. Neuron 42:253-268.

Ilmoniemi RJ, Virtanen J, Ruohonen J, Karhu J, Aronen HJ, Näätänen R, Katila T (1997) Neuronal responses to magnetic stimulation reveal cortical reactivity and connectivity. Neuroreport 8:3537-3540.

Jeong J (2004) EEG dynamics in patients with Alzheimer's disease. Clin Neurophysiol 115:1490-1505.

Kähkönen S, Komssi S, Wilenius J, Ilmoniemi RJ (2005a) Prefrontal transcranial magnetic stimulation produces intensity-dependent EEG responses in humans. Neuroimage 24:955-960.

Kähkönen S, Komssi S, Wilenius J, Ilmoniemi RJ (2005b) Prefrontal TMS produces smaller EEG responses than motor-cortex TMS: implications for rTMS treatment in depression. Psychopharmacology (Berl) 181:16-20.

Komssi S, Kähkönen S, Ilmoniemi RJ (2004) The effect of stimulus intensity on brain responses evoked by transcranial magnetic stimulation. Hum Brain Mapp 21:154-164.

Laufs H, Krakow K, Sterzer P, Eger E, Beyerle A, Salek-Haddadi A, Kleinschmidt A (2003) Electroencephalographic signatures of attentional and cognitive default modes in spontaneous brain activity fluctuations at rest. Proc Natl Acad Sci U S A 100:11053-11058.

Llinás RR, Ribary U, Jeanmonod D, Kronberg E, Mitra PP (1999) Thalamocortical dysrhythmia: a neurological and neuropsychiatric syndrome characterized by magnetoencephalography. Proc Natl Acad Sci U S A 96:15222-15227.

Llinás RR, Choi S, Urbano FJ, Shin HS (2007) Gamma-band deficiency and abnormal thalamocortical activity in P/Q-type channel mutant mice. Proc Natl Acad Sci U S A 104:17819-17824.

Mantini D, Perrucci MG, Del Gratta C, Romani GL, Corbetta M (2007) Electrophysiological signatures of resting state networks in the human brain. Proc Natl Acad Sci U S A 104:13170-13175.

Massimini M, Ferrarelli F, Huber R, Esser SK, Singh H, Tononi G (2005) Breakdown of cortical effective connectivity during sleep. Science 309:2228-2232.

Massimini M, Ferrarelli F, Esser SK, Riedner BA, Huber R, Murphy M, Peterson MJ, Tononi G (2007) Triggering sleep slow waves by transcranial magnetic stimulation. Proc Natl Acad Sci U S A 104:8496-8501.

Michel CM, Lehmann D, Henggeler B, Brandeis D (1992) Localization of the sources of EEG delta, theta, alpha and beta frequency bands using the
FFT dipole approximation. Electroencephalogr Clin Neurophysiol 82:38-44.

Narici L, Romani GL (1989) Neuromagnetic investigation of synchronized spontaneous activity. Brain Topogr 2:19-30.

Niedermeyer E (1999) The normal EEG of the waking adult. In: Electroencephalography (Niedermeyer E, Lopes da Silva F, eds). Baltimore: Williams and Wilkins.

Pastor MA, Artieda J, Arbizu J, Marti-Climent JM, Peñuelas I, Masdeu JC (2002) Activation of human cerebral and cerebellar cortex by auditory stimulation at $40 \mathrm{~Hz}$. J Neurosci 22:10501-10506.

Paus T, Sipila PK, Strafella AP (2001) Synchronization of neuronal activity in the human primary motor cortex by transcranial magnetic stimulation: an EEG study. J Neurophysiol 86:1983-1990.

Rager G, Singer W (1998) The response of cat visual cortex to flicker stimuli of variable frequency. Eur J Neurosci 10:1856-1877.

Regan D (1989) Human brain electrophysiology: evoked potentials and evoked magnetic fields in science and medicine New York: Elsevier.

Silberstein S (1995) Steady-state visually evoked potentials, brain resonances and cognitive processes. In: Neocortical dynamics and human EEG rhythms (Nunez P, ed). New York: Oxford UP.

Soininen H, Reinikainen K, Partanen J, Mervaala E, Paljärvi L, Helkala EL, Riekkinen P Sr (1992) Slowing of the dominant occipital rhythm in electroencephalogram is associated with low concentration of noradrenaline in the thalamus in patients with Alzheimer's disease. Neurosci Lett 137:5-8.

Tamraz J, Comair Y (2000) Atlas of regional anatomy of the brain using MRI. Berlin: Springer.

Thielscher A, Kammer T (2002) Linking physics with physiology in TMS: a sphere field model to determine the cortical stimulation site in TMS. Neuroimage 17:1117-1130.

Tinguely G, Finelli LA, Landolt HP, Borbély AA, Achermann P (2006) Functional EEG topography in sleep and waking: state-dependent and state-independent features. Neuroimage 32:283-292.

Tononi G, Srinivasan R, Russell DP, Edelman GM (1998) Investigating neural correlates of conscious perception by frequency-tagged neuromagnetic responses. Proc Natl Acad Sci U S A 95:3198-3203.

Van Der Werf YD, Sadikot AF, Strafella AP, Paus T (2006) The neural response to transcranial magnetic stimulation of the human motor cortex. II. Thalamocortical contributions. Exp Brain Res 175:246-255.

Virtanen J, Ruohonen J, Näätänen R, Ilmoniemi RJ (1999) Instrumentation for the measurement of electric brain responses to transcranial magnetic stimulation. Med Biol Eng Comput 37:322-326.

von Stein A, Chiang C, König P (2000) Top-down processing mediated by interareal synchronization. Proc Natl Acad Sci U S A 97:14748-14753.

Wassermann EM (1998) Risk and safety of repetitive transcranial magnetic stimulation: report and suggested guidelines from the International Workshop on the Safety of Repetitive Transcranial Magnetic Stimulation, June 5-7, 1996. Electroencephalogr Clin Neurophysiol 108:1-16. 